\title{
Efficacy and Safety of Once Daily Fluticasone Furoate Nasal Spray for Treatment of Irritant (Non-allergic) Rhinitis
}

\author{
Pongsakorn Tantilipikorn ${ }^{1}$, Sanguansak Thanaviratananich ${ }^{2}$, Supinda Chusakul $^{3}$, \\ Suwat Benjaponpitak ${ }^{4}$, Supranee Fooanant ${ }^{5}$, Chalermchai Chintrakarn ${ }^{6}$, \\ Orathai Jirapongsananuruk ${ }^{7}$, Nualanong Visitsunthorn ${ }^{7}$, Tom Toler ${ }^{8}$, \\ Laura Sutton ${ }^{8}$, Wei $\mathrm{Wu}^{8}$ and Laurie Lee ${ }^{*}, 8$
}

\author{
${ }^{I}$ Department of Otorhinolaryngology, Siriraj Hospital, Mahidol University, Bangkok, Thailand \\ ${ }^{2}$ Department of Otorhinolaryngology, Khon Kaen University, Khon Kaen, Thailand \\ ${ }^{3}$ Department of Otolaryngology, Chulalongkorn University, Bangkok, Thailand \\ ${ }^{4}$ Division of Allergy \& Immunology, Department of Pediatrics, Faculty of Medicine, Ramathibodi Hospital, Mahidol \\ University, Bangkok, Thailand \\ ${ }^{5}$ Department of Otolaryngology, Chiang Mai University, Chiang Mai, Thailand \\ ${ }^{6}$ Department of Otolaryngology, Faculty of Medicine, Ramathibodi Hospital, Mahidol University, Bangkok, Thailand \\ ${ }^{7}$ Allergy \& Immunology Division, Department of Pediatrics, Siriraj Hospital, Mahidol University, Bangkok, Thailand \\ ${ }^{8}$ GlaxoSmithKline, Research Triangle Park, NC, USA
}

\begin{abstract}
Introduction: The safety and efficacy of fluticasone furoate nasal spray (FFNS) for the symptoms of seasonal and perennial allergic rhinitis have been previously demonstrated in several clinical studies. The objective of this pilot study was to compare the efficacy and safety of FFNS $110 \mathrm{mcg}$ once daily with placebo in patients with irritant (nonallergic) rhinitis triggered predominantly by air pollution.

Methods: This was a randomized, double-blind, placebo-controlled, 4-week study of subjects in Thailand with irritant rhinitis who received either FFNS $110 \mathrm{mcg}(\mathrm{N}=53)$ or placebo $(\mathrm{N}=49)$ once-daily. Subjects with a $\geq 2$ year history of air pollution as their predominant rhinitis trigger, negative skin test to local seasonal/perennial allergens, positive histamine skin test, and normal sinus radiograph were enrolled if they met minimum reflective total nasal symptom score (rTNSS) $\geq 4.5$ (maximum=9) and a nasal congestion score of $\geq 2$ (maximum $=3$ ). All were assessed for nasal eosinophilia at study entry and completion. Air quality was monitored throughout the study. The primary efficacy endpoint was the mean change from baseline over the treatment period in daily rTNSS, the average of the morning and evening rTNSS. The key secondary measure was the mean change from baseline over the entire treatment period in morning pre-dose instantaneous total nasal symptom score (iTNSS).

Results: The Air Quality Index (AQI) during the study generally did not reach unhealthy levels. Baseline daily rTNSS scores were similar between treatment groups ( $F F N S=6.7$; placebo=6.4). The least square mean change from baseline in rTNSS was -2.17 and -2.10 for FFNS and placebo, respectively, with a difference of -0.065 ( $p=0.845$ ). Gradual improvements were seen in both treatment groups for iTNSS; however, the treatment difference $(-0.075)$ was not statistically significant $(\mathrm{p}=0.827)$. Additionally, there were no statistically significant differences between treatment groups for AM and PM rTNSS, individual nasal symptoms, daily reflective, AM and PM reflective and AM pre-dose instantaneous total ocular symptom scores (TOSS) or individual ocular symptom scores. Nasal cytology at baseline found more than two-thirds of subjects had $<20 \%$ eosinophils. Adverse events were few and similar between groups and noted as mild in intensity.

Conclusion: Subjects receiving FFNS had similar improvement to placebo in their rhinitis symptoms. The lack of a treatment effect may be in part due to the overall good air quality present throughout the study or an insufficient dose or duration of FFNS. The safety findings showed FFNS $110 \mathrm{mcg}$ once daily to be well tolerated.
\end{abstract}

Keywords: Fluticasone furoate, irritant, rhinitis.

*Address correspondence to this author at the GlaxoSmithKline, 5 Moore Drive, Research Triangle Park, NC 27709, USA; Tel: (919) 483-7909; Fax: (919) 483-4300; E-mail: laurie.a.lee@gsk.com

\section{INTRODUCTION}

Rhinitis is a common disorder affecting $10 \%$ to $30 \%$ of all adults and as many as $40 \%$ of children [1-5] with 
symptoms that include rhinorrhea, congestion, sneezing, post-nasal drip, and ocular and nasal itching. When assessed immunologically, rhinitis is divided into allergic (IgEdependent) and non-allergic (IgE-independent) etiologies. Research into the cause and treatment of allergic rhinitis is abundant in the medical literature; however, nonallergic rhinitis (NAR) research has been constrained by a meager understanding of the pathophysiology and inconsistent categorization of the various NAR conditions and their definitions. This disparity in the medical science creates a unique problem in clinical practice as non-allergic rhinitis may occur in up to $25 \%$ of rhinitis sufferers and contribute to symptoms in as many as $60 \%$ ('mixed rhinitis') [6-8].

NAR is a general term for chronic perennial nasal conditions that can be categorized into many forms, for instance the imbalance of neural innervations, vasomotor rhinitis (VMR), or irritant rhinitis. The majority of patients with NAR are thought to have VMR or non-allergic rhinitis with eosinophilia syndrome (NARES). Using immunologic and cytologic features to further categorize NAR, irritant rhinitis may be considered a non-inflammatory, non-allergic subclass of VMR [7]. Airborne irritants and changes in the weather are considered by some to be the two primary categories of triggers for VMR. Airborne irritants include chemicals, glues, solvents, perfumes, offensive odors, pollution, and smoke. Weather and temperature changes include fluctuations in temperature, humidity, barometric pressure, or air movements $[9,10]$.

An alternative classification scheme for NAR based on etiology considers irritant-induced rhinitis a discrete entity from VMR with at least five cited causes or types of irritants: dry air, gustatory (food-induced), bright light, occupational, and air pollution [7,11]. This scheme is in alignment with the US Food and Drug Administration's (FDA) position that VMR is predominantly triggered by changes in the weather and irritant rhinitis is a distinct form of NAR [12]. The FDA's position formed the basis for selecting a study population of subjects with irritant rhinitis whose symptoms are primarily triggered by respiratory irritants.

Air pollution is a common irritant most people have encountered at some time in occupational exposure, the home, or the outdoor environment. The majority of air pollution is caused by the burning of fossil fuels and the resultant pollutants of nitrogen oxides, carbon monoxide, sulfur dioxides and various particulate matter [11]. Atmospheric breakdown of nitrogen oxides to ozone, in particular, has been a significant problem in the warmer months necessitating daily reporting of levels as a warning for the elderly and people with respiratory disease. Studies of human exposure to air pollution have been limited for ethical reasons by the known toxicity of air contaminants, the particle concentrations necessary to emulate poor conditions, and the duration of experimental exposures that needed to correlate with actual exposure. However, data from small studies of various pollutants, some in concentrations found in urban western areas, show pollutants may alter nasal function and produce nasal symptoms [13,14]. Moreover, chronic rhinitis, regardless of immunologic origin, is a potent risk factor for the development of asthma.
Fluticasone furoate nasal spray (FFNS) has been studied extensively in allergic disease and found to demonstrate consistent efficacy and safety in seasonal as well as perennial allergic rhinitis. Two studies of VMR triggered by weather/temperature changes were conducted to assess the efficacy of FFNS in non-allergic disease [9]. However, neither study found a significant difference from placebo for any measure of efficacy. The authors concluded that VMR may be a distinct disease variant which is refractory to treatment with intranasal steroids. The goal of this pilot study was to investigate the efficacy of FFNS in a different subset of NAR, subjects with symptoms attributable to air pollution occurring in the urban areas of Thailand.

\section{MATERIALS AND METHODOLOGY}

\section{Patients and Study Design}

This 4-week, Phase IIa, randomized, double-blind, placebo-controlled, parallel-group, multicenter study (FFR111158) was conducted in Thailand at 7 study centers from March 2008 through February 2009. The protocol and one protocol amendment were approved by the individual centers' ethics committees. The study was conducted in accordance with good clinical practice and the Declaration of Helsinki with written informed consent being obtained from each subject or the subject's parent/guardian if the subject was $<18$ years old. The study consisted of a 2 -week screening period, a 4-week treatment period, and a 3- to 5day follow-up telephone call after treatment ended. The study design is shown in Fig. (1).

\section{Assessments}

Subjects 12 years of age and older who satisfied the entry criteria and did not meet the exclusion criteria (nasal obstruction, septal perforation, recent nasal surgery, nasal infections) entered a screening period for a minimum of 7 days and a maximum of 14 days. Inclusion criteria required subjects to identify air pollution as the predominant trigger that made their rhinitis symptoms worse. To establish this, subjects completed an irritant rhinitis trigger questionnaire at Visit 1 where they were first asked to select their predominant trigger from three types of irritants (air pollution, wind/temperature triggers, and strong odors), then identify specific triggers within those three groups.

Inclusion criteria also included a negative skin prick test to local seasonal and perennial allergens, a positive histamine control skin prick test, and a normal sinus radiograph (Waters view) to rule out sinusitis. A nasal swab was also performed using a Rhino-probe ${ }^{\mathrm{TM}}$ Curette for the purpose of evaluating nasal cytology for treatment stratification if the subject met randomization criteria. Samples were analyzed by Quest Diagnostics Clinical Trials Laboratory (Van Nuys, California).

During the screening period, subjects scored three nasal (rhinorrhea, nasal congestion, post-nasal drip) and three ocular symptoms (eye itching/burning, eye tearing/watering, and eye redness) each morning and evening on a paper diary card in order to determine eligibility for randomization. Symptom ratings were performed with respect to the presence/severity of symptoms at the moment in the morning just prior to dosing (instantaneous rating, [i]) and over the previous 12 hours (reflective rating, [r]). A reflective 


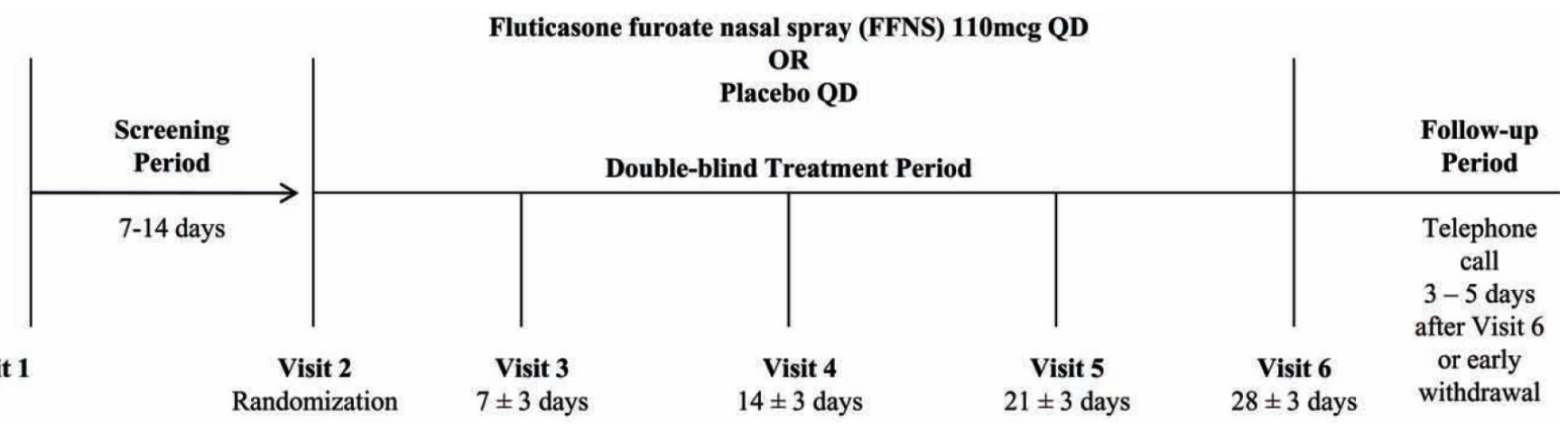

Fig. (1). Study design.

assessment for the previous 12 hours was also conducted in the evening (PM). The three individual reflective nasal and ocular symptom scores were combined to obtain an AM and PM reflective total nasal symptom score (AM rTNSS, PM rTNSS) and total ocular symptom score (AM rTOSS, PM rTOSS), respectively. These two scores were then averaged to obtain a daily reflective total nasal (daily rTNSS) and total ocular symptom score (daily rTOSS). Similarly, the instantaneous pre-dose morning scores were summed to obtain an instantaneous predose TOSS (AM pre-dose iTOSS). The rating scale for symptom assessment was based on a widely used, 4-point categorical scale of 0 to 3 (none, mild, moderate, severe) [15].

Randomization criteria at Visit 2 required subjects to have an average of $\geq 4.5$ (maximum $=9$ ) for the last 8 reflective TNSS assessments, an average of $\geq 2$ (maximum $=3$ ) for the last 8 reflective nasal congestion assessments, and an $80 \%$ compliance with their diary card entries over the span of their screening period. Subjects who fulfilled the randomization criteria were randomly assigned in a 1:1 ratio to receive either FFNS $110 \mathrm{mcg}$ once daily or vehicle placebo nasal spray once daily with the first dose being administered in the clinic following device demonstration. Subjects were stratified into two groups based on nasal cytology: those with eosinophils constituting $\geq 5 \%$ or $<5 \%$ of nasal white blood cells.

Subjects continued their nasal and ocular ratings throughout the remainder of the study and returned to the clinic weekly for four weeks (Visits 3 to 6) during the treatment period. Subjects were instructed not to take any anti-allergy/anti-rhinitis medication during the screening or treatment periods of the study and no rhinitis rescue medication was allowed during the study. Medications known to produce allergy symptoms, such as congestion, were not allowed. Use of face masks (e.g., used for protection from air pollution), continuous positive airflow pressure, saline nasal sprays and lavages, eye drops, and local, herbal and homeopathic treatments were also prohibited. Daily air quality data from Thailand's Environmental Pollution Control website was obtained for the period of the study along with air quality data for the previous 12 months. Subjects documented the amount of time they spent indoors and outdoors, including how much time was spent in air conditioned environments. Study drug compliance, any medical conditions they experienced, and any concomitant medications they took were also recorded on diary cards.

\section{Statistical Analysis}

As this was a small pilot study, the study was not powered to test for a treatment difference. A sample size of 100 subjects (50 per arm) was used to provide an estimated mean treatment difference between FFNS and placebo for the primary efficacy endpoint, the mean change from baseline in the daily rTNSS over Weeks 1 through 4 . With this sample size, the precision for the estimated mean treatment difference (half the width of the $95 \%$ confidence interval) was expected to be $\leq 0.666$, assuming a standard deviation of 1.7 which was based on the previous VMR studies where the same assessment ratings were used [9].

The primary efficacy analysis was performed using analysis of covariance, adjusting for baseline value, baseline nasal eosinophil count, age, and gender.

\section{RESULTS}

\section{Patients}

A total of 102 subjects were randomized and received at least one dose of study treatment. Mean compliance with treatment over the 4 week treatment was $94.1 \%$ for FFNS and $98.6 \%$ for placebo. Demographic and baseline characteristics were similar between the treatment and placebo groups (Table 1). As the study was conducted in Thailand, all subjects were Asian and the majority classified themselves as being of South East Asian heritage.

Table 1. Patient Demography and Baseline Characteristics

\begin{tabular}{|l|c|c|c|}
\hline & $\begin{array}{c}\text { FFNS 110 mcg } \\
\text { QD (N=53) }\end{array}$ & $\begin{array}{c}\text { Placebo } \\
\mathbf{( N = 4 9 )}\end{array}$ & $\begin{array}{c}\text { Total } \\
(\mathbf{N}=\mathbf{1 0 2})\end{array}$ \\
\hline \hline Mean Age, years (SD) & $37.1(12.78)$ & $35.9(10.89)$ & $36.6(11.87)$ \\
Range & $12-58$ & $18-64$ & $12-64$ \\
\hline Gender, n (\%) & & & \\
Female & $33(62)$ & $37(76)$ & $70(69)$ \\
Male & $20(38)$ & $12(24)$ & $32(31)$ \\
\hline
\end{tabular}

Table 2 shows the specific triggers noted by the subjects in each group. Eighty-seven percent (87\%) of subjects enrolled in the study reported all 3 categories of triggers.

Air quality data during the study was obtained from Thailand's Pollution Control Department website, which is operated by the Ministry of Natural Resources and the Environment. The study was originally planned to coincide with 
Table 2. Trigger Questionnaire Responses

\begin{tabular}{|c|c|c|c|}
\hline \multicolumn{4}{|l|}{ Air Pollution } \\
\hline Outside dust & $45(85)$ & $42(86)$ & $87(85)$ \\
\hline Exhaust (cars, trucks, buses) & $43(81)$ & $40(82)$ & $83(87)$ \\
\hline Industrial gases/fumes & $22(42)$ & $16(33)$ & $38(37)$ \\
\hline \multicolumn{4}{|l|}{ Weather/Temperature Triggers } \\
\hline Any & $49(92)$ & $46(94)$ & $95(93)$ \\
\hline Windy days & $9(17)$ & $15(31)$ & $24(24)$ \\
\hline Cold days & $36(68)$ & $29(59)$ & $65(64)$ \\
\hline Hot summer days & $12(23)$ & $9(18)$ & $21(21)$ \\
\hline \multicolumn{4}{|l|}{ Strong Odor Triggers } \\
\hline Any & $51(96)$ & $44(90)$ & $95(93)$ \\
\hline Smoke (tobacco/burning items) & $47(89)$ & $35(71)$ & $82(80)$ \\
\hline Perfumes & $16(30)$ & $9(18)$ & $25(25)$ \\
\hline Cosmetics & $9(17)$ & $4(8)$ & $13(13)$ \\
\hline Cleaning products/detergents/soaps & $7(13)$ & $13(27)$ & $20(20)$ \\
\hline Paint fumes or paint products & $30(57)$ & $23(47)$ & $53(52)$ \\
\hline Hairspray & $16(30)$ & $17(35)$ & $33(32)$ \\
\hline Fumes from domestic space heating & $18(34)$ & $13(27)$ & $31(30)$ \\
\hline
\end{tabular}

the dry winter months when greater pollution is typically present. However, delays in site ethical approvals resulted in a March 2008 start for the study. The wet season in Thailand typically runs from June to October. The Air Quality Index (AQI) ranged as high as 110 on daily ratings, however, at no time did it exceed a weekly mean AQI of 100, the lower end of the range defined as unhealthy for sensitive groups (Fig. 2). On average, subjects in both treatment groups spent approximately six hours daily inside. In addition, subjects spent at least eight hours each day outside; however, subjects in the FFNS group spent $0.5-1.5$ hours longer outside compared with subjects in the placebo group.

\section{Efficacy}

\section{Primary Endpoint}

The primary efficacy measure was the mean change from baseline in the reflective total nasal symptom score (rTNSS) over the entire treatment period (weeks 1 - 4). At baseline, the mean daily rTNSS was similar between the two groups (Table 3). A gradual improvement was seen in both treatment groups over each week in the study, with slightly greater improvements in subjects receiving FFNS (Fig. 3). However, the treatment difference over Weeks 1 through 4 was not statistically significant (Table $\mathbf{3}$ ).

\section{Secondary Endpoints}

A key secondary efficacy measure was the mean change from baseline over weeks 1 through 4 in morning pre-dose instantaneous total nasal symptom score (iTNSS). As with the daily rTNSS, a gradual improvement was seen in both treatments groups; however, the mean treatment difference (0.075 ) was not statistically significant $(\mathrm{p}=0.827)$. Additionally, there were no statistically significant differences between treatment groups for AM and PM rTNSS, individual nasal symptoms (rhinorrhea, nasal congestion or post-nasal drip), the reflective and instantaneous scores for TOSS, or the individual ocular symptom scores (eye itching/burning, eye tearing/watering or eye redness).

\section{Nasal Cytology}

Subjects' nasal smears were assessed to determine the percentage of eosinophils for stratification purposes. Slightly more than half of the subjects demonstrated $<5 \%$ eosinophils in their nasal smear at baseline (Table 4). Mean percent eosinophils were comparable at baseline for both treatment groups. Mean percent eosinophils were lower for FFNS at the end of the study compared with placebo.

\section{Tolerability}

Twenty-one subjects (40\%) in the FFNS group and 18 subjects $(37 \%)$ in the placebo group reported adverse events. Most events were described as mild. The most commonly reported events $(\geq 3 \%$ incidence and more common than placebo) were cough ( $6 \%$ vs $2 \%)$, migraine (4\% vs $2 \%)$, nasal ulcer (4\% vs 2\%) and epistaxis (4\% vs 0$)$ for FFNS and placebo, respectively. All adverse events reported, except one event of increased transaminase, were deemed unrelated 


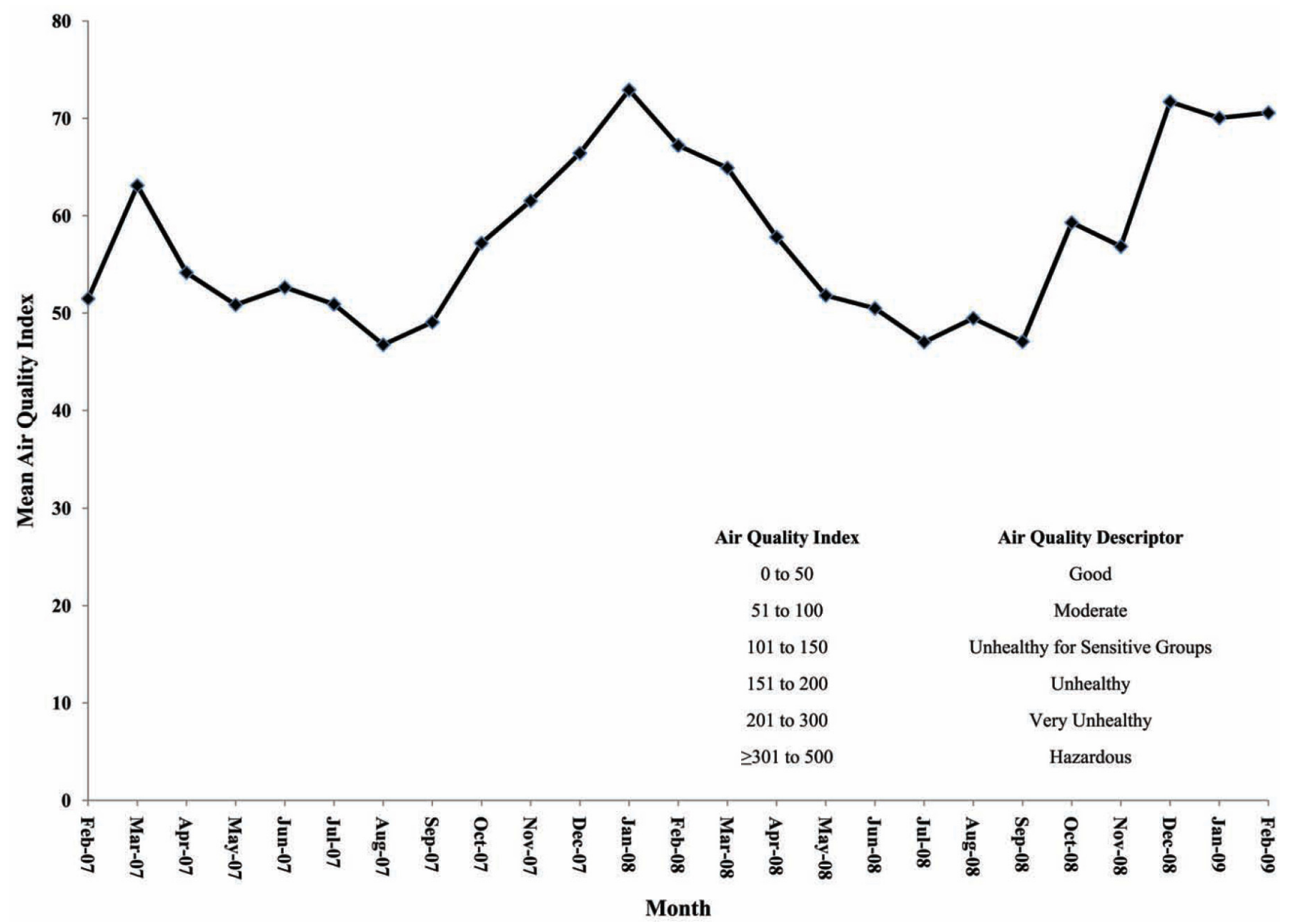

Fig. (2). Mean weekly level of overall AQI (one year prior to and over the study period).

Table 3. Weekly and Overall Mean Change from Baseline in Daily Reflective Total Nasal Symptom Score (rTNSS)

\begin{tabular}{|l|c|c|}
\hline & $\begin{array}{c}\text { FFNS 110 mcg QD } \\
\mathbf{( N = 5 3 )}\end{array}$ & $\begin{array}{c}\text { Placebo } \\
\mathbf{( N = 4 9 )}\end{array}$ \\
\hline \hline Baseline & & \\
Mean (SE) & $6.7(0.17)$ & $6.4(0.17)$ \\
Weeks 1 - 4 & $4.4(0.25)$ & $4.4(0.24)$ \\
Mean (SE) & & \\
\hline Week 1 & $-1.6(0.22)$ & $-1.3(0.19)$ \\
Mean change (SE) & 53 & 49 \\
Week 2, n & $-2.1(0.26)$ & $-1.8(0.28)$ \\
Mean change (SE) & 51 & 47 \\
Week 3, n & $-2.5(0.29)$ & $-2.4(0.28)$ \\
Mean change (SE) & 49 & 46 \\
Week 4, n & $-2.9(0.30)$ & $-2.8(0.29)$ \\
Mean change (SE) & & \\
\hline Weeks 1 - 4 & $-2.2(0.24)$ & $-2.0(0.24)$ \\
Mean change (SE) & $-2.17(0.23)$ & $-2.10(0.25)$ \\
Least squares mean change (SE) & -0.065 & \\
Least squares mean difference & 0.845 & \\
p-value & & \\
\hline
\end{tabular}

to study drugs. In this event, the subject who was receiving FFNS experienced an increase in alanine aminotransferase (ALAT) from 8 to $97 \mathrm{U} / \mathrm{L}$ and a rise in aspartate aminotransferase (ASAT) from 13 to $44 \mathrm{U} / \mathrm{L}$. This event was noticed at the final treatment visit. The subject was followed and the elevations were noted to be resolved 24 days later.

\section{DISCUSSION}

In this randomized, placebo-controlled, pilot study which evaluated Thai subjects with a narrowly-defined condition, irritant rhinitis primarily triggered by air pollution, FFNS did not demonstrate efficacy in reducing the nasal symptoms of this condition. The lack of efficacy for FFNS in this study may be attributable to several factors. While the study attempted to obtain a pure irritant rhinitis population triggered by air pollution, $\geq 90 \%$ of subjects also reported specific types of weather/temperature and strong odor triggers. The actual study population aligns closely with the aforementioned classification of NAR where irritant rhinitis is a subset of VMR, a condition characterized by nonspecific nasal hyperreactivity in response to non-immunologic stimuli such as temperature, relative humidity, strong odors and other airborne irritants [7].

This study highlights that the characteristics of this clinical population do not align with classification schemes that distinguish subtypes of NAR based on triggers alone. 


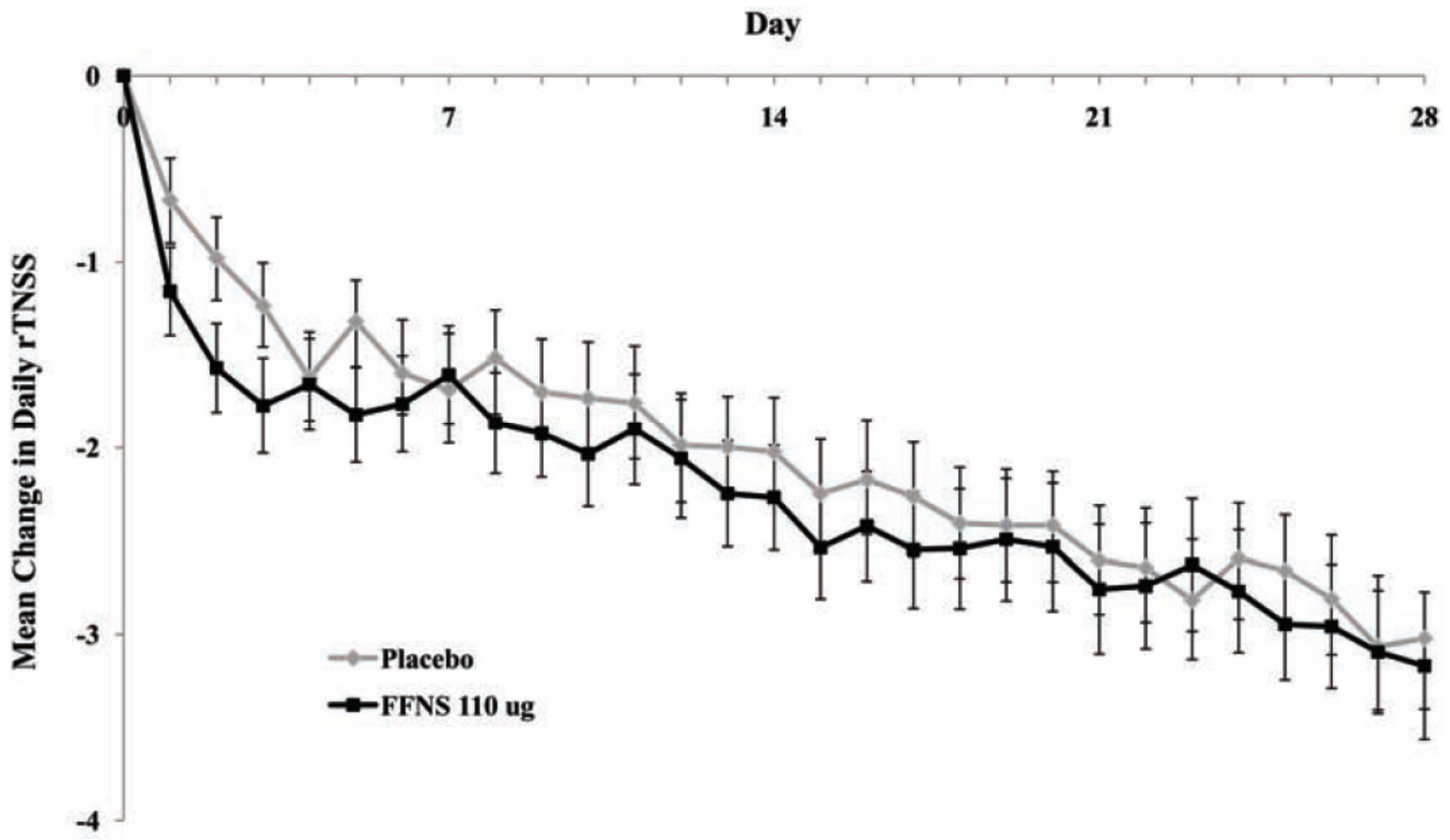

Fig. (3). Daily mean change from baseline in daily reflective total nasal symptom score over weeks $1-4$.

Patients with NAR are likely to have several triggers and may, in addition, also have allergic rhinitis. Two previous randomized controlled trials of NAR found FFNS ineffective in subjects selected for weather/temperature-sensitive VMR [9]. Thus, results from this pilot study are not completely surprising in that the study population identified air pollution as their primary trigger, but nearly all subjects had other non-allergic triggers, including changes in the weather. Our study results do not rule out the possibility that FFNS may be effective for the symptoms of irritant rhinitis in subjects with isolated airborne irritant triggers or at a different dose, frequency of administration, or longer duration than shown to be effective for allergic rhinitis. In addition, confirmation of the subjects' responses on the trigger questionnaire with nasal provocation challenges may have helped to further refine the study population.

The decision to conduct this pilot study in Thailand was based on historical air quality history, availability of air quality monitoring, the close proximity of investigative sites, and a temperate climate to avoid weather/temperature changes thought to precipitate VMR. Thailand currently uses the US Environmental Protection Agency (EPA) air quality assessment scale of 0 to 500 where an air quality index (AQI) value between 0 and 100 corresponds to an air quality standard of good (0-50) to moderate (51-100) which are considered satisfactory. For the majority of the study, weekly air quality fell within the moderate range at all sites, with some sites even demonstrating weekly levels below 50 . Given the better than expected air quality, this study does not rule out an effect of FFNS in irritant rhinitis where exposure to air pollution is ensured.
Although subjects were required to meet minimum symptom criteria prior to randomization, those with more severe symptoms may have declined to participate due to the study requirement of withholding medications during the screening period. The moderate to severe symptom criteria for inclusion may have inadvertently permitted enrollment of chronic irritant rhinitis patients who consistently sustain a moderate irritation from the air pollution, when present, but never exacerbate to symptom levels high enough where a treatment difference versus placebo could be demonstrated. The possibility for this effect was increased in this study since the AQI never reached unhealthy levels consistently high enough to precipitate severe symptoms. The difficulty in demonstrating a treatment difference was also compounded by the moisturizing benefit of the placebo nasal spray administered just prior to subjects starting their day, a recurring influence in trials conducted with topically administered placebos. A placebo benefit is evidenced by the fact subjects in both treatment groups improved.

NAR subtypes are often distinguished by the presence or absence of nasal eosinophils but nasal cytology data from patients with irritant rhinitis triggered by respiratory irritants is lacking. The eosinophil cytology for the current study found over $50 \%$ of subjects in both groups had $5 \%$ or fewer eosinophils at baseline with nearly two-thirds having fewer than $20 \%$. The observation that greater than half the subjects corresponded to a non-inflammatory population may provide an explanation for the inability to demonstrate a treatment difference with FFNS because eosinophilia has been suggested as a predictor of the effectiveness of intranasal corticosteroid therapy [12]. 
Table 4. Summary of Percent Eosinophils

\begin{tabular}{|c|c|c|c|c|}
\hline & \multicolumn{2}{|c|}{ FFNS $110 \mathrm{mcg}$ QD $(\mathrm{N}=53)$} & \multicolumn{2}{|c|}{ Placebo (N=49) } \\
\hline & Baseline & Endpoint & Baseline & Endpoint \\
\hline Mean (SD) & $14.2(22.04)$ & $5.7(14.30)$ & $14.9(22.03)$ & $12.0(22.42)$ \\
\hline Median & 4.0 & 0.0 & 2.5 & 0.5 \\
\hline \multirow[t]{2}{*}{ Minimum - Maximum } & $0-84$ & $0-70$ & $0-87$ & $0-97$ \\
\hline & \multicolumn{4}{|c|}{ n (\%) } \\
\hline$<5 \%$ & $29(55)$ & $44(83)$ & $28(57)$ & $30(61)$ \\
\hline $5 \%-<10 \%$ & $4(8)$ & $1(2)$ & $2(4)$ & $4(8)$ \\
\hline $10 \%-<15 \%$ & $5(9)$ & $2(4)$ & $2(4)$ & $3(6)$ \\
\hline $15 \%-<20 \%$ & $3(6)$ & $1(2)$ & $2(4)$ & $3(6)$ \\
\hline$\geq \mathbf{2 0} \%$ & $12(23)$ & $5(9)$ & $15(31)$ & $9(18)$ \\
\hline
\end{tabular}

If the symptomatology is elicited by a neurogenic mechanism in response to the pollution rather than an inflammatory process, FFNS would not be expected to have a therapeutic effect for subjects with irritant rhinitis. A neurogenic mechanism for NAR is supported by the findings that both parasympathetic and sympathetic neurons affect epithelial, vascular, as well as glandular functions in the nose [10].

\section{CONCLUSION}

The dose and duration of treatment with FFNS for seasonal and perennial allergic rhinitis was not effective for this population of subjects with irritant rhinitis caused predominantly by air pollution. These results, combined with two previous randomized controlled trials demonstrating FFNS to be ineffective in subjects selected for weather/temperature-sensitive VMR, suggest that intranasal corticosteroids may not be effective in subtypes of NAR where eosinophilic inflammation is not a major component [9]. Additional studies should investigate the pathophysiology of irritant rhinitis, other treatments or treatment combinations, including non-steroidal options, as well as other endpoints including biomarkers or change in nasal mucosa responsiveness to methacholine.

\section{TRIAL REGISTRATION}

www.clinicaltrials.gov; NCT00730756.

\section{ABBREVIATIONS}

ALAT = Alanine aminotransferase

$\mathrm{AM}=$ Morning

AQI = Air quality index

ASAT $=$ Aspartate aminotransferase

EPA $=$ Environmental protection agency

FFNS = Fluticasone furoate nasal spray

iTNSS $=$ Instantaneous total nasal symptom score

NAR $=$ Non-allergic rhinitis

NARES $=$ Non-allergic rhinitis with eosinophilia syndrome

= Evening
rTNSS $=$ Reflective total nasal symptom score

TNSS $=$ Total nasal symptom score

TOSS $=$ Total ocular symptom score

VMR = Vasomotor rhinitis

\section{CONFLICT OF INTEREST}

Drs. Tantilipikorn, Thanaviratananich, Chusakul, Benjaponpitak, Fooanant, Chintrakarn, Jirapongsananuruk and Visitsunthorn are investigators for GlaxoSmithKline.

Drs. Toler, Sutton, $\mathrm{Wu}$ and Lee are full-time employees of GlaxoSmithKline.

\section{ACKNOWLEDGEMENTS}

The authors would like to thank Chaweewan Bunnag, MD (Siriraj Hospital, Mahidol University; Bangkok, Thailand) for her participation in the study and comments on the manuscript. The authors would also like to thank Edward E. Philpot, MD for his contributions to the design and implementation of the study as well as data analysis.

This study was funded by GlaxoSmithKline.

\section{REFERENCES}

[1] Settipane RA. Rhinitis: a dose of epidemiological reality. Allergy Asthma Proc 2003; 24: 147-54.

[2] Druce H. Allergic and nonallergic rhinitis. In: Middleton E, Reed CE, Ellis EF, Adkinson NF Jr, Yunginger W, Busse WW, Eds. Allergy principles and practice. 5th ed. St Louis: Mosby-Year Book 1998; pp. 1005-16.

[3] Settipane RJ, Hagy GW, Settipane GA. Long-term risk factors for developing asthma and allergic rhinitis: a 23 -year follow-up study of college students. Allergy Proc 1994; 15: 21-25.

[4] Varjonen E, Kalimo K, Lammintausta K, Terho P. Prevalence of atopic disorders among adolescents in Turku, Finland. Allergy 1992; 47: 243-48.

[5] Smith JM. A five-year prospective survey of rural children with asthma and hay fever. J Allergy 1971; 47: 23-30.

[6] Togias A, Naclerio RM, Proud D, et al. Studies on the allergic and nonallergic nasal inflammation. J Allergy Clin Immunol 1988; 81: 782-90.

[7] Settipane RA, Lieberman P. Update on nonallergic rhinitis. Ann Allergy Asthma Immunol 2001; 86:494-507.

[8] Molgaard E, Thomsen SF, Lund T, et al. Differences between allergic and nonallergic rhinitis in a large sample of adolescents and adults. Allergy 2007; 62: 1003-7. 
[9] Jacobs R, Lieberman P, Kent E, et al. Weather/temperaturesensitive vasomotor rhinitis may be refractory to intranasal corticosteroid treatment. Allergy Asthma Proc 2009; 30: 120-27.

[10] Fokkens WJ. Thoughts on the pathophysiology of non-allergic rhinitis. Curr Allergy Asthma Rep 2002; 2: 203-09.

[11] Riedl, MA. The effect of air pollution on asthma and allergy. Curr Allergy Asthma Rep 2008; 8: 139-46.

[12] Settipane RA, Charnock DR. Epidemiology of rhinitis: Allergic and nonallergic. In: Baraniuk JN, Shusterman D, Eds. Nonallergic rhinitis. New York: Informa Healthcare 2007.
[13] Shusterman D. Environmental nonallergic rhinitis. In: Baraniuk JN, Shusterman D, Eds. Nonallergic rhinitis. New York: Informa Healthcare 2007.

[14] Riechelmann H, Rettinger G, Weschta M, et al. Effects of lowtoxicity particulate matter on human nasal function. J Occup Environ Med 2003; 45: 54-60.

[15] FDA Draft Guidance for Allergic Rhinitis: Clinical Development Programs for Drug Products, April 2000. Assessed June 21, 2010 http://www.fda.gov/cder/guidance/2718dft.pdf

(C) Tantilipikorn et al.; Licensee Bentham Open.

This is an open access article licensed under the terms of the Creative Commons Attribution Non-Commercial License (http://creativecommons.org/licenses/ by-nc/3.0/) which permits unrestricted, non-commercial use, distribution and reproduction in any medium, provided the work is properly cited. 